Iran Journal of Nursing (IJN)

Vol 34, No. 129, Apr 2021: 39- 49

\title{
The Effect of Peer Education on the Anxiety of Patients with Thalassemia Major: A Quasi-experimental Study
}

\author{
Mohammad Sadegh Sargolzaei ${ }^{1}$, Alice Khachian ${ }^{2}$, Mahnaz Seyedoshohadaee ${ }^{3}$, \\ Hamid Haghani ${ }^{4}$
}

\begin{abstract}
Background \& Aims: Thalassemia major (TM) is one of the most common chronic genetic disorders in today's world. The number of patients with thalassemia in the world is estimated at 240 million and is reported in 60 countries. Iran is located in the thalassemia belt and has about 26,000 patients with thalassemia major. Patients with chronic diseases such as thalassemia have to undergo continuous medical treatment throughout their lives and their survival depends on these treatments. The treatment protocol affects different aspects of these patients' lives and can make them anxious. Therefore, any measures taken to reduce the anxiety of these patients are very important. Educational intervention can be a method for improving the quality of life in patients with chronic diseases. Nowadays, the educational methods that engage learners in their learning process have acquired attention. One of the methods to educate patients is peer education which has been effective in promoting their health status. In this educational approach, patients share their experiences about their common disease. Having worked in the thalassemia ward, the researcher realized the importance of peer education as a scientific and codified method for efficient education of these patients. Moreover, considering the clinical observation of psychological problems in patients with thalassemia major and also the lack of continuous and reliable research in this field, the present study aimed to evaluate the effect of peer education on the anxiety experienced by patients with thalassemia major.
\end{abstract}

Materials \& Methods: This one-group, quasi-experimental study was conducted at Imam Khomeini Hospital in Zabol between March and July 2020 on 50 participants, who were selected via continuous sampling from the patients with the thalassemia major based on the inclusion and exclusion criteria of the study. Data were collected using a demographic questionnaire exploring participants' age, sex, residency, occupation, level of education, economic status, parents' familial relationship, the frequency of blood transfusions per month, another family member with the same disease, the type of iron-chelating agent, regular drug consumption, and history of other diseases. In addition, the valid and reliable Beck Anxiety Inventory (BAI) was used to measure anxiety. The study began with the selection of eligible peers educators based on inclusion criteria. Finally, 2 patients with thalassemia major (a man and a woman) who obtained higher scores were selected as peer educators, who were then trained by the researcher in 3 sixty-minute educational sessions within one week. The educational content included explanations about research objectives, the importance and benefits of peer education, educational concepts, communication skills including attention to verbal and non-verbal behaviors, active listening skills, and anxiety control concepts. The educational content was taught through an interactive lecture using audio-visual aids, and question and answer. Based on the training given by the researcher and the experiences expressed by the peer educators, their final evaluation was conducted through a role-play. After training the peer educators, the pre-test was conducted. Afterwards, an anxiety management training program

\footnotetext{
1. MS in Nursing, School of Nursing and Midwifery, Iran University of Medical Sciences, Tehran, Iran

2. Nursing Care Research Center, Department of Internal-Surgical Nursing, School of Nursing and Midwifery, Iran University of Medical Sciences, Tehran, Iran

${ }^{3}$. Nursing Care Research Center, Department of Internal-Surgical Nursing, School of Nursing and Midwifery, Iran University of Medical Sciences, Tehran, Iran (Corresponding author) Tel: 02143651606 Email: seyedoshohadaee.m@iums.ac.ir

4. Department of Biostatistics, School of Health, Iran University of Medical Sciences, Tehran, Iran
} 
was presented to the participants with thalassemia major which included the definition of anxiety, its symptoms and its role in exacerbating the disease, strategies to reduce and control anxiety, revision of beliefs, and negative and anxious attitudes. The training program, with emphasis on the peer educators' experiences, was conducted over two 60-minute sessions in two consecutive hours. Participants were placed in groups of 6 to 7 patients. Each group was trained separately by a peer in a training class in the thalassemia ward. After the training sessions, the educational booklet was given to all participants of the study. One month after the intervention, the participants took the post-test. The present research was approved by the Ethics Committee of Iran University of Medical Sciences. The researcher adhered to the ethical policies such as obtaining informed consent from the participants and their voluntary participation. Data analysis was performed in SPSS version 16 using descriptive statistics (mean and standard deviation) and inferential statistics (paired-sample t-test).

Results: The mean age of the participants was $24.5 \pm 4.24$ years. In this study, most participants were women, had a diploma, and were unemployed. Moreover, the parents of most of the patients had familial relationships. The mean score of anxiety was $17.73 \pm 11.63$ in the pretest, which reduced to $17.07 \pm 11.32$ in the posttest. The results of paired t-test indicated no significant differences in the pretest and posttest scores of anxiety in the patients with the thalassemia major $(\mathrm{t}=0.788 ; \mathrm{P}=0.434)$.

Conclusion: According to the results, the peer education program did not have any positive effects on the anxiety level of the patients with thalassemia major. Considering the nature of educational interventions, these patients may benefit from concurrent anxiety-reducing interventions such as relaxation exercises and music therapy. The findings also suggest that future researches use other assessment tools, broader educational content, and a control group to assess anxiety in patients with thalassemia major. Furthermore, it is recommended that researchers compare the effects of peer training with other anxiety management training methods used for these patients.

Keywords: Peer Group, Anxiety, Thalassemia Major, Patient Education

\section{Conflict of Interest: No}

How to Cite: Sargolzaei MS, Khachian A, Seyedoshohadaee M, Haghani H. The Effect of Peer Education on the Anxiety of Patients with Thalassemia Major: A Quasi-experimental Study. Iran Journal of Nursing. 2021; 34(129): 39-49.

Received: 9 Jan 2021

Accepted: 10 Apr 2021 


\title{
تأثير آموزش همتا بر اضطراب بيماران مبتلا به تالاسمى مازور: يك مطالعه نيمه تجربى
}

\author{
محمدصادق سر كلزائى '، آليس خاجيان '، مهناز سيدالشهرايى '، حميد حقانىع
}

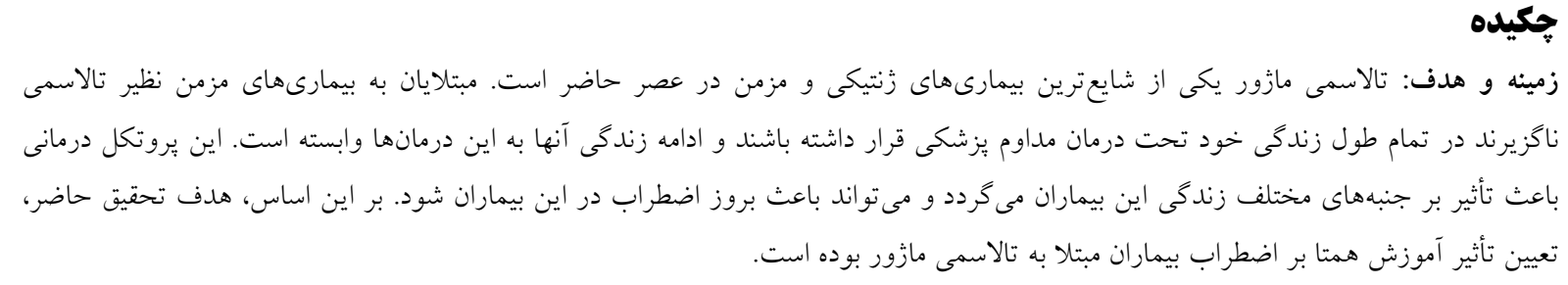

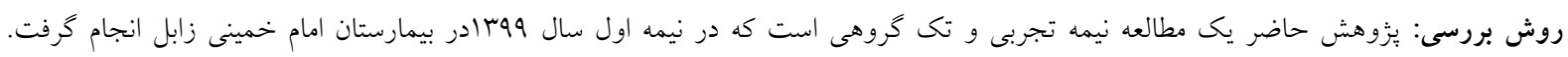

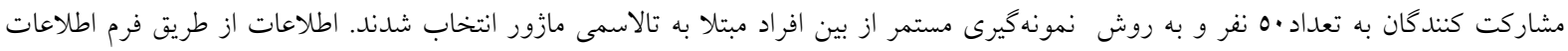

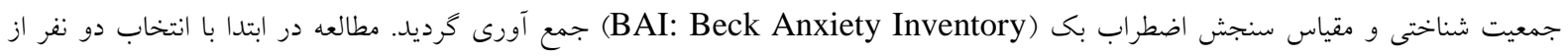

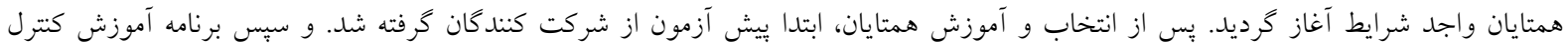

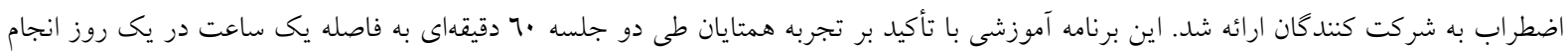

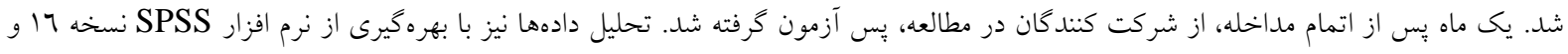

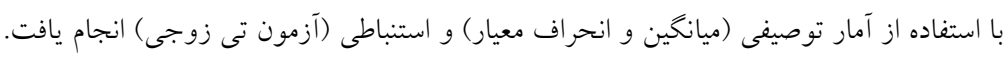

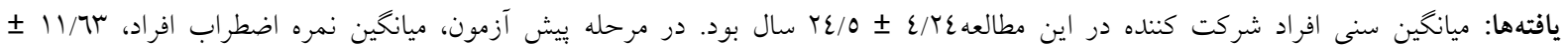

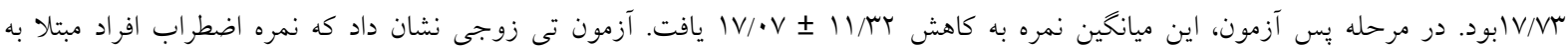

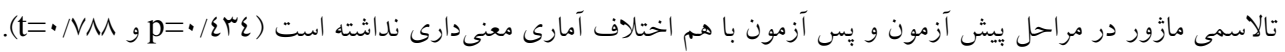

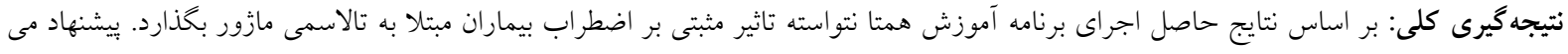

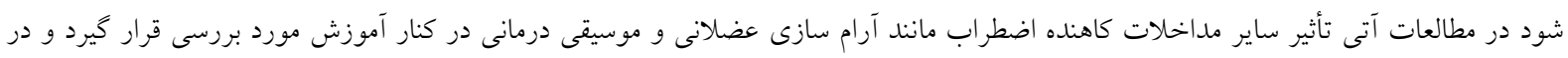

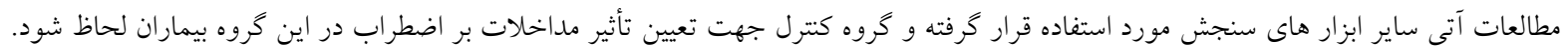

كليد وازهها: گروه همتا، اضطراب، تالاسمى مازور، آموزش به بيمار

$$
\text { تعارض منافع: وجود ندارد. }
$$

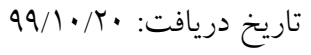

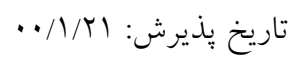

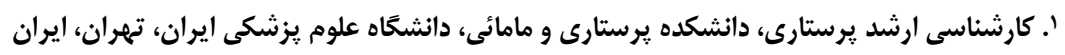

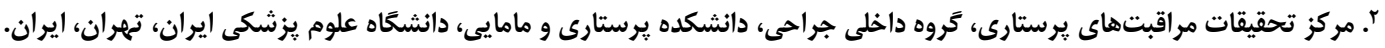

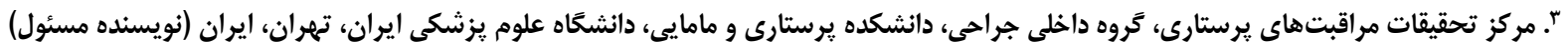

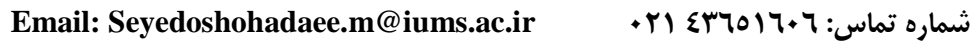

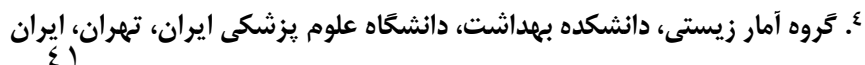


اضطراب، افسردگى و اختلالات جسمانى است(10.17). بر اساس نتايج مطالعهاى ميزان اين مشكلات در در بيماران

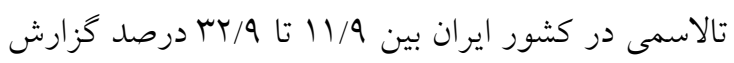

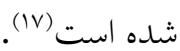
بيماران مبتلا به تالاسمى مازور همانند همه بيمارىهاى

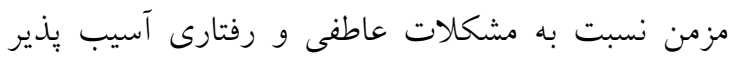
هستند (1). در واقع يكى از مسائلى كه اين بيماران با آن مآن

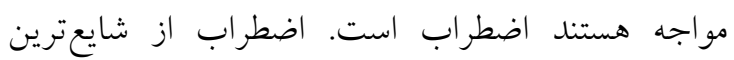

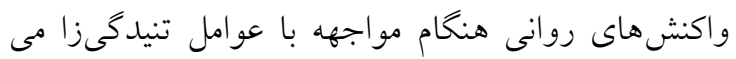

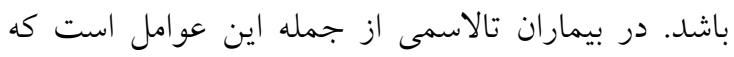
تنيدكى ايجاد كرده و سبب فراخوانى بسيارى از واكنش بنش

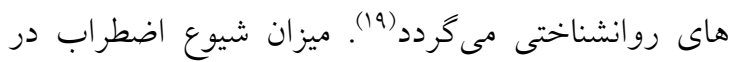
بيماران تالاسمى در مطالعه ماهرى و همكاران (•r) كه درد

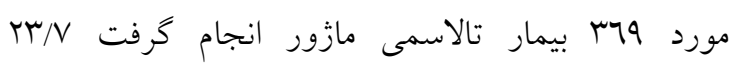

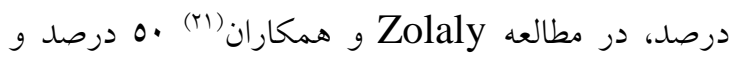

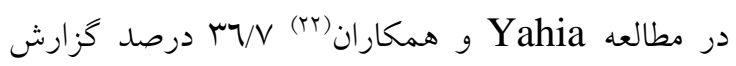

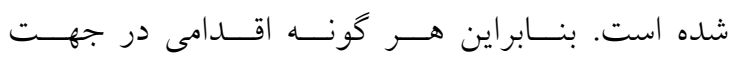

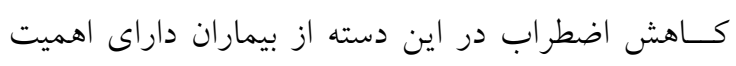

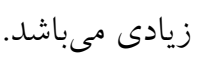
از آموزش به بيمار مىتوان بانده به عنوان يكى راهكار جهت

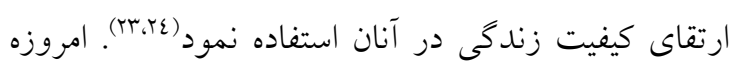

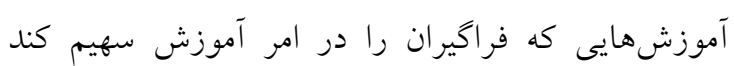

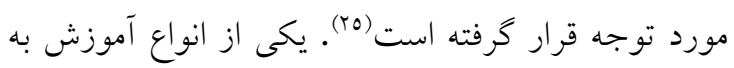

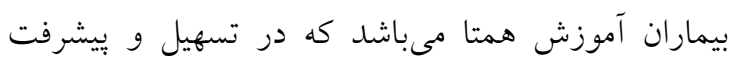

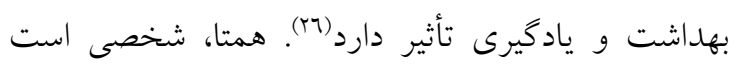
متعلق به كروه اجتماعى يكسان، كه مشاهده كنند كان باور

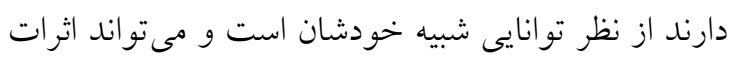

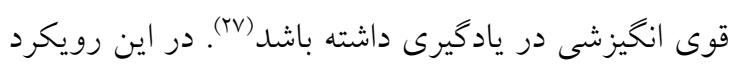

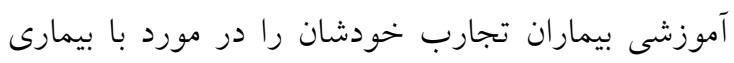

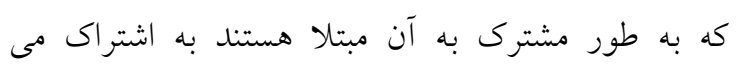

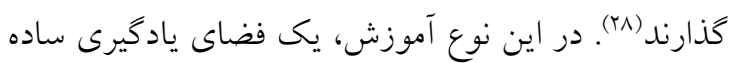

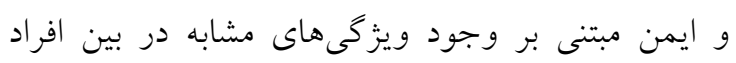

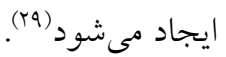

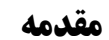

تالاسمى به عنوان شايعترين اختلال همو گلوبينوياتى ارثى

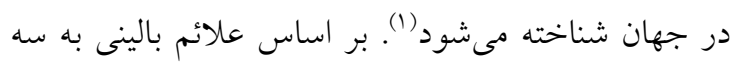
دسته مينور، اينترمديا و مازور تقسيم بندى مى شود (r).

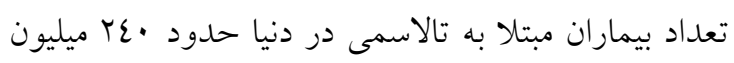

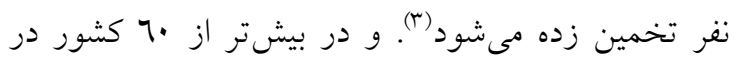

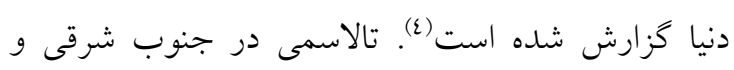

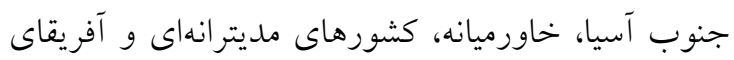

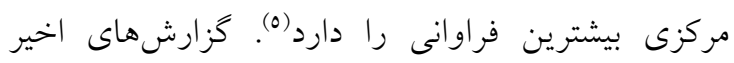
نشان مىدهد كه 1/0 درصد از جمعيت جهان حامل جنين

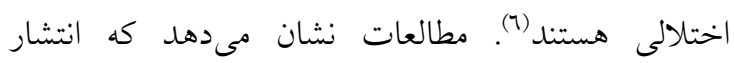
تالاسمى مازور تهديدى جدى براى كشورهاى كم درآمد

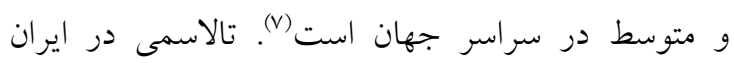
شايعترين بيمارى زنتيكى است و حدود سه ميليون نفر

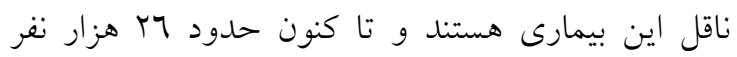

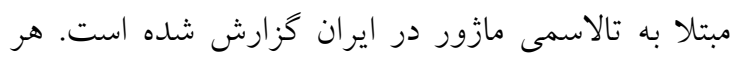

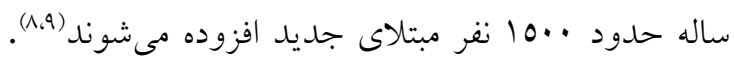
آمارها نشان مىدهد كه در استانهاى خوزستان، بوشهر،

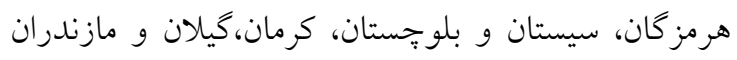
تا •ا درصد از مردم حامل زنهاى اين بيمارى هستند (.1).

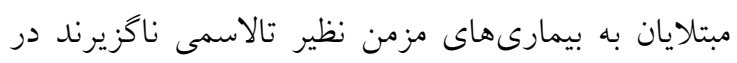

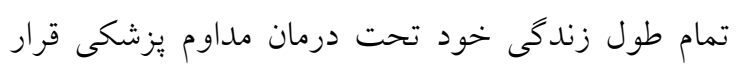

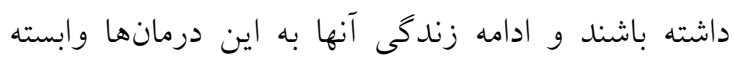

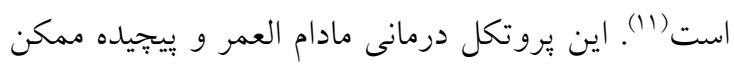

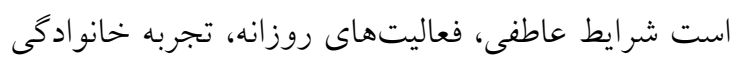

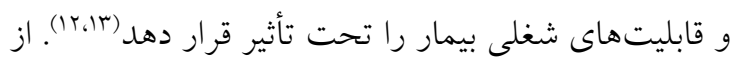
سوى ديخر تزريق مكرر خون سبب اضافه بار آهن و

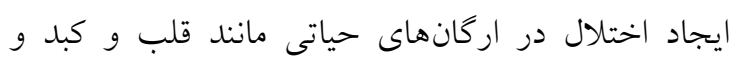
ايجاد بيمارىهاى همراه مىشود و زمينه را براى تهديد

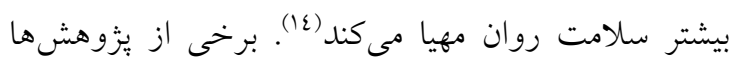
نشان مىدهند كه •N درصد مبتلايان به تالاسمى مازور حداقل يك مشكل روان شناختى را دارند. بيشترين

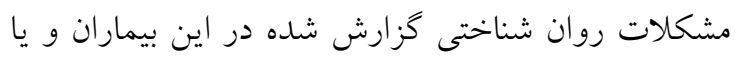

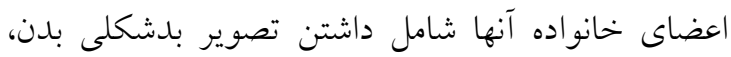


بيمار بود. حجم نمونه با در نظر كرفتن سطح اطمينان

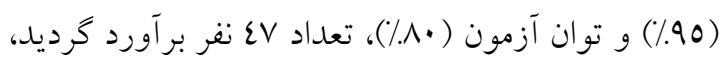

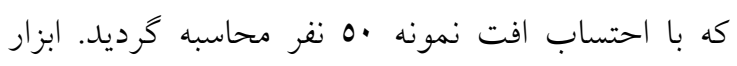

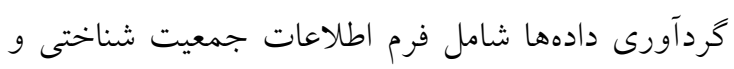

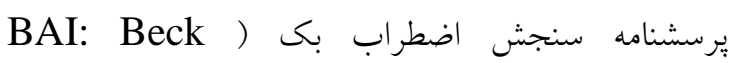
(Anxiety Inventory شامل سن، جنس، محل سكونت، شغل، سطح تحصيلات،

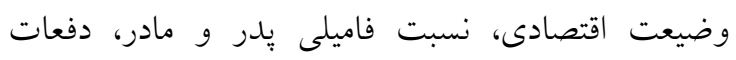

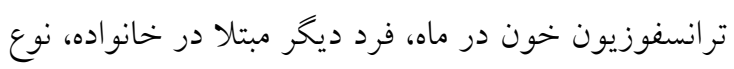

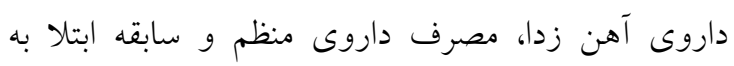
بيمارى ديخر بود.

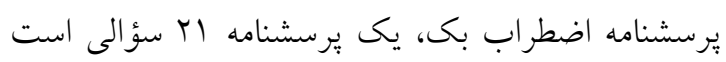

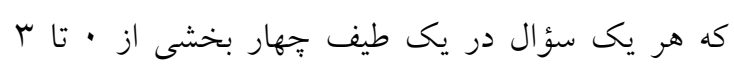

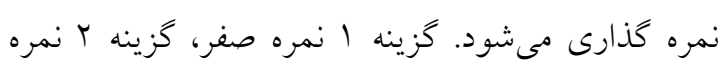

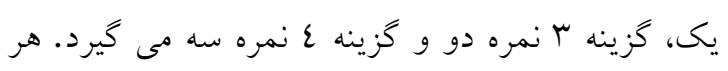
يك از مادهاى آزمون يكى از علائم شايع اضطراب

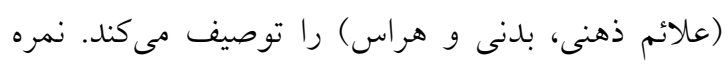

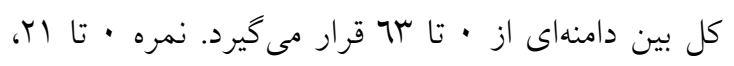

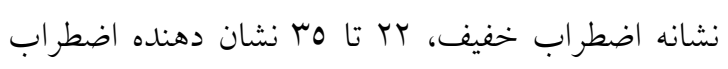

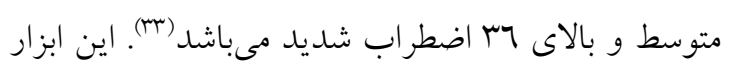

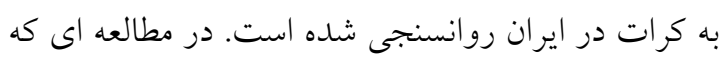

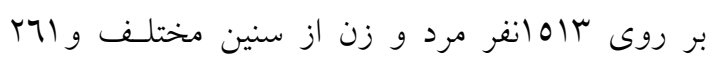
بيمار اضطرابى مراجعسه كنـــه بـهـ درمانخاهها و مراكز

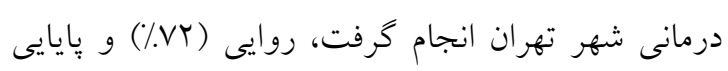

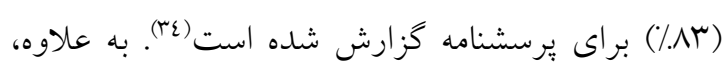

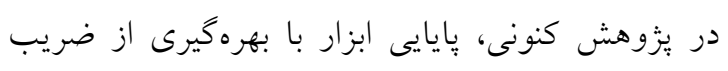

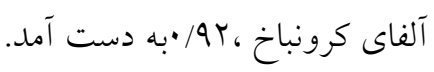

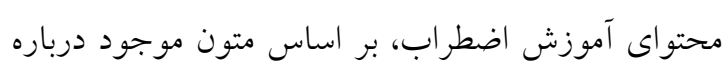

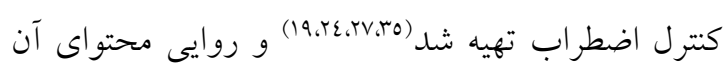
به تأييد ينج نفر از اعضاى هيأت علمى يرستارى، رسيد.

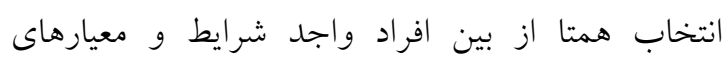

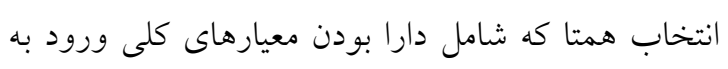

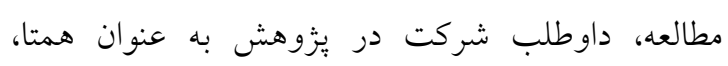

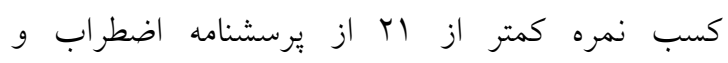

در مطالعات زيادى بر سودمندى رابطه بين بيمار و همتا

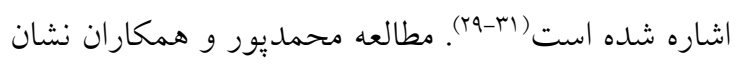

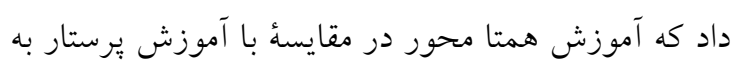
طور معنى دارى باعث كاهش اضطراب بيماران مبتلا به

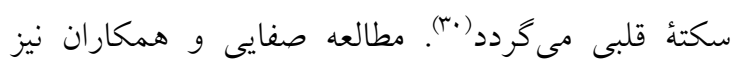
نشان دهنده بهبود خودمراقبتى در بيماران تالاسمى در اثر فردئ

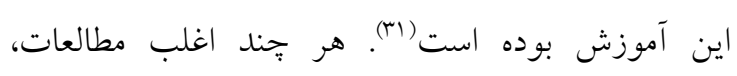
كوياى تأثير مثبت آموزش همتا در افراد بودهاند، اما نتايج يكى مرور نظاممند حاكى از آن است كه در همه موارد،

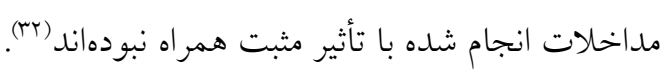
ثزوهشگر در مدت كار در بخش تالاسمى، متوجه خلأ

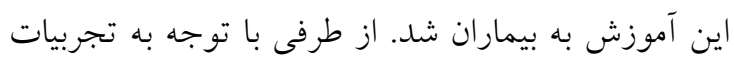

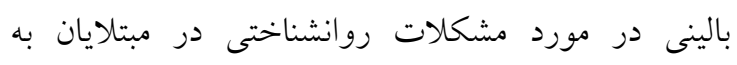

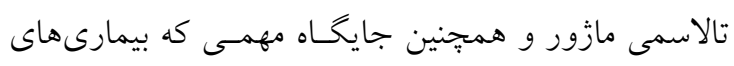
مزمن در مطالعات سلامت عمومى و مداخلههاى سازنده دارند و اهميت مقوله سلامت روان و نقش تعيين كننده آن در بهبود كيفيت روند درمان، تداوم آن و همنينين

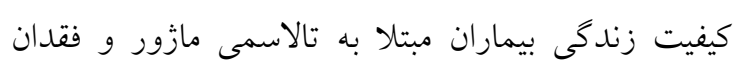

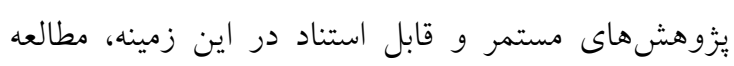

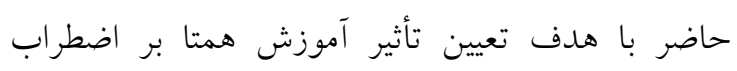
بيماران مبتلا به تالاسمى مازور انجام يافت.

\section{روش بررسى}

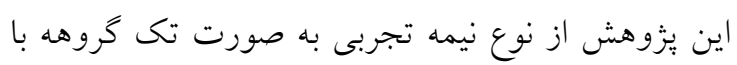

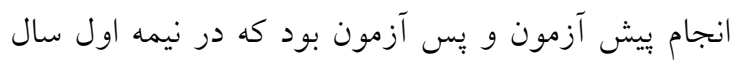

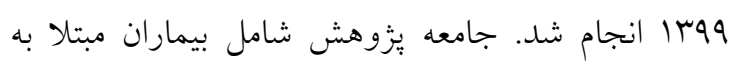
تالاسمى مازور مراجعه كننده به بخش تالاسمى بيمارستان

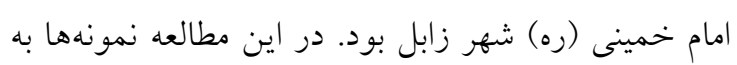
صورت مستمر نمونه گيرى شدند. معيارهاى ورود بيماران

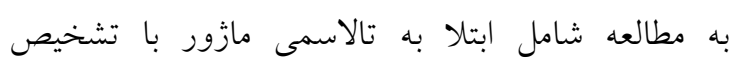

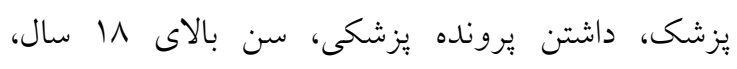
نداشتن مشكل شناختى و معلوليت جسمى و داشتن سواد

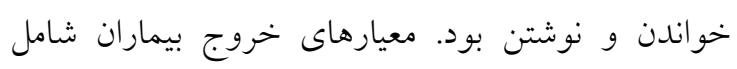

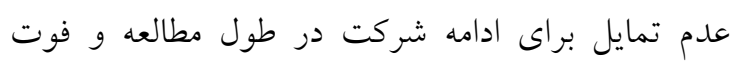


افراد در بخش تالاسمى جهت ترانسفوزيون خون و بر

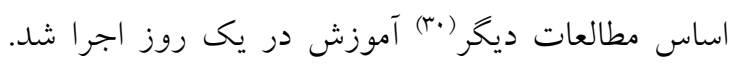
جلسه اول آموزشى شامل معارفه همتا با ساير نمونهها،

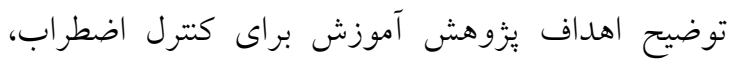
تعريف اضطراب و علائم و نقش آن در تشديد بيمارى و ورون

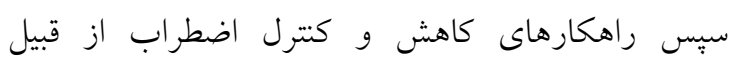
تمرينات تنفسى، تصوير سازى ذهنى و ورزش، تجديد

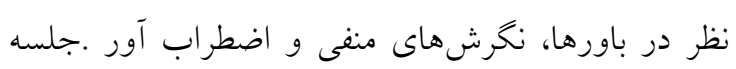

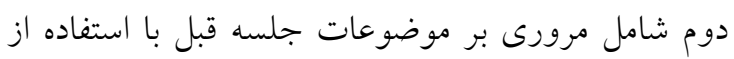

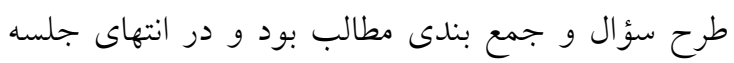

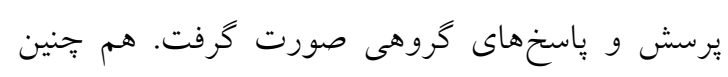

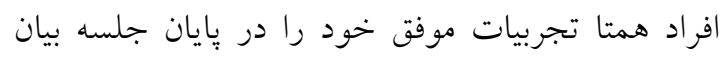

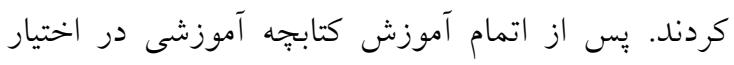

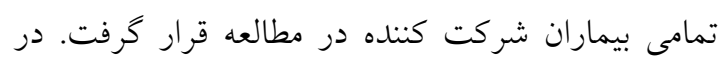

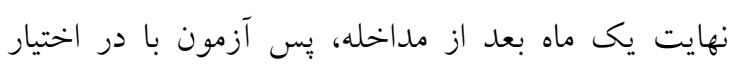

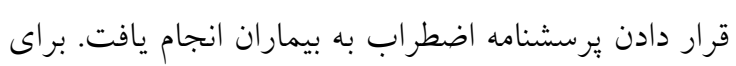

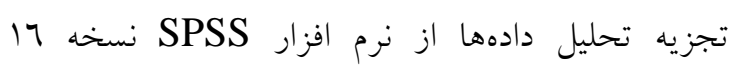

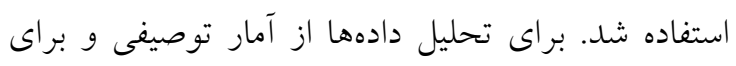

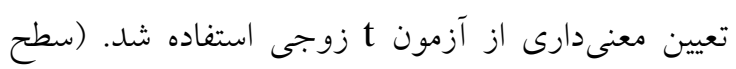

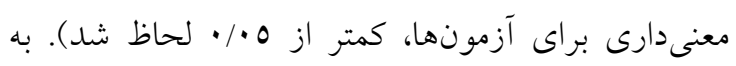

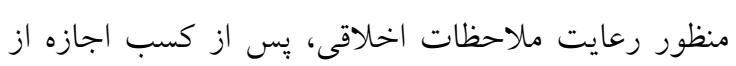

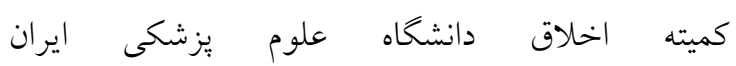
(IR.IUMS.REC.1398.910) ورود به بيمارستان امام خمينى زابل اخذ شد. همجِنين، به

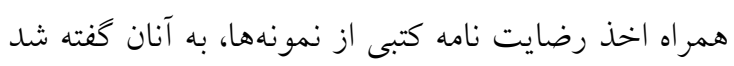

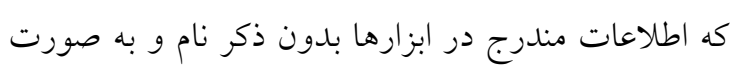

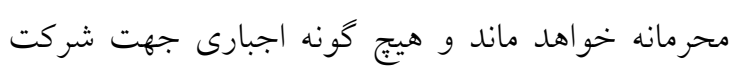
در بيزوهش وجود ندارد.

\section{يافتهها}

بيشتر شركت كنندكان در اين مطالعه، زن بودند. ميانخين

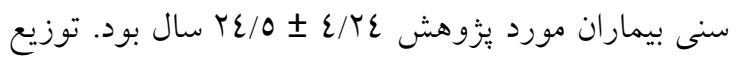

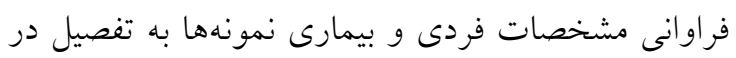

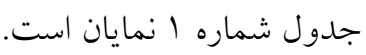

تحصيلات دييلم و بالاتر بود انجام كرفت. در نهايت دو نفر (يك آقا و يكى خانم)كه امتياز بيشترى نسبت به به بقيه

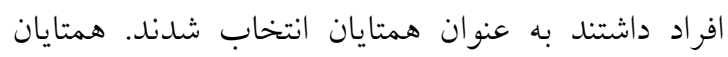

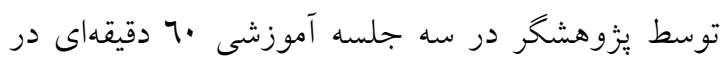

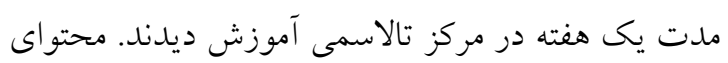

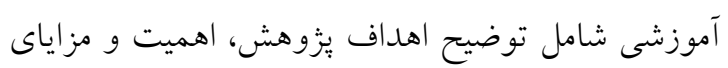
آموزش همتا، مفاهيم مورد استفاده براى آموزش، مهارت آندات

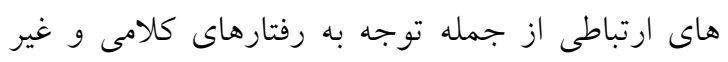

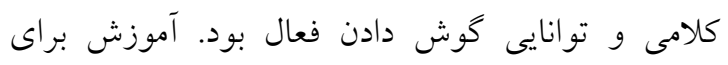
كنترل اضطراب شامل تعريف اضطراب و علائم و نقش بنش

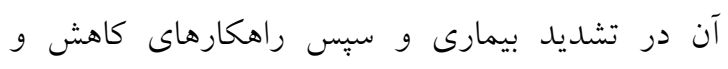

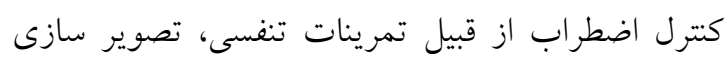

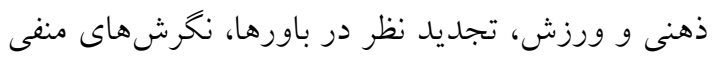

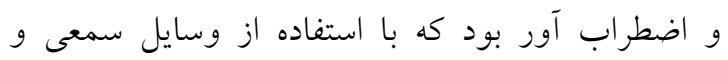

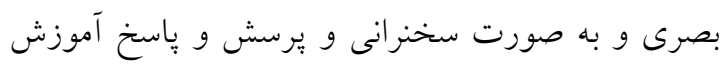

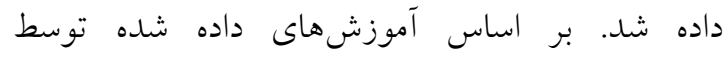

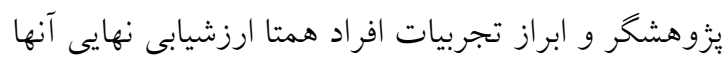

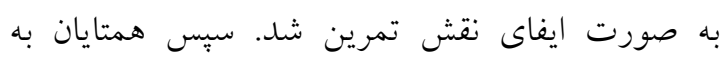

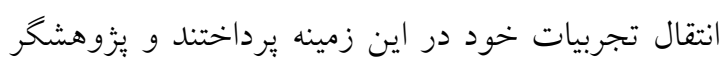

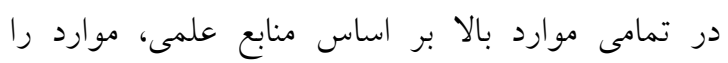

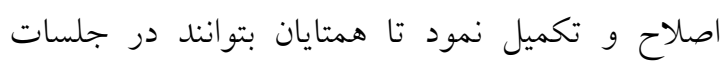

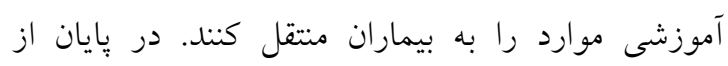

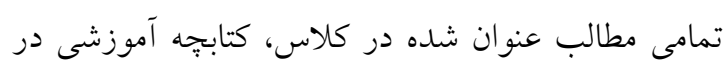

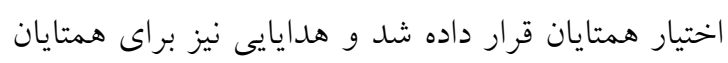

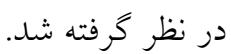
يس از كسب رضايت آكاهانه از بيماران جهت شركت در

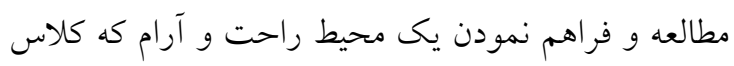

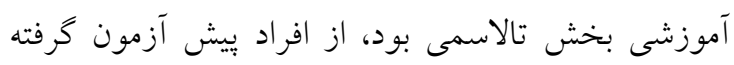

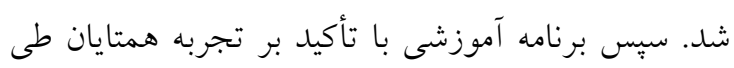

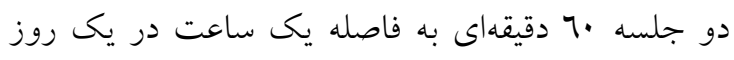

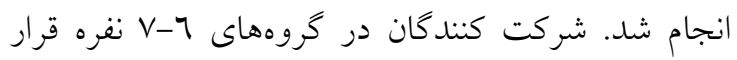

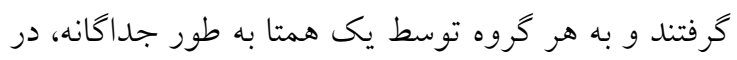

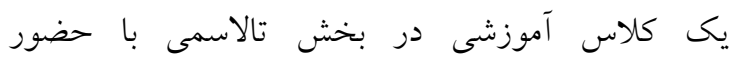

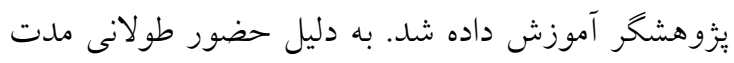


جدول شماره (: توزيع فراوانى مشخصات جمعيت شناختى بيماران مبتلا به تالاسمى مازور

\begin{tabular}{|c|c|c|c|c|c|c|c|}
\hline \multirow{2}{*}{ درصد } & \multirow{2}{*}{ تعداد } & \multicolumn{2}{|c|}{ مشخصات فردى } & \multirow{2}{*}{ درصد } & \multirow{2}{*}{ تعداد } & \multicolumn{2}{|c|}{ مشخصات فردى } \\
\hline & & شهر & محل سكونت & & & $111-19$ & \\
\hline 1. & 0 & اطراف شهر & & $\mu_{\Lambda}$ & 19 & $r \cdot-r \varepsilon$ & سن (سال) \\
\hline$\varepsilon\rceil$ & $r$ & ا روستا & & $r$. & 10 & ro-rq & \\
\hline 1.. & 0. & جمع & & 11 & 9 & $r \cdot-r \varepsilon$ & \\
\hline 7. & $r \cdot$ & فاميلى & & $1 .$. & 0. & جمع كل & \\
\hline \multirow[t]{2}{*}{$\varepsilon$. } & r. & غير فاميلى & نسبت يدر و مادر & \multicolumn{2}{|c|}{$r \varepsilon / 0 \pm \varepsilon / r \varepsilon$} & انحر اف معيار 土 ميانخين & \\
\hline & & & & \multicolumn{2}{|c|}{$\mid \Lambda-r \varepsilon$} & بيشينه- كمينه & \\
\hline $1 \ldots$ & 0. & جمع & & 07 & rA & زن & \\
\hline 1. & 0 & يك بار & دفعات ترانسفوزيون & $\varepsilon \varepsilon$ & rr & مرد & 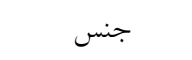 \\
\hline 17 & $\varepsilon r$ & دو بار & خون در ماه & $1 \ldots$ & 0. & جمع كل & \\
\hline$\varepsilon$ & r & بدون بِاسخ & & $r \varepsilon$ & IT & ابتدايى & \\
\hline $1 .$. & 0. & جم & & rr & 17 & متوسطه & \\
\hline m & 11 & بله & & m & 11 & دييلم & سطح تحصيلات \\
\hline$\pi$ & r & 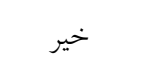 & فرد ديغر مبتلا در & 7 & $r$ & بالاتر از دييلم & \\
\hline r & 1 & بدون ياسخ & خانواده & r & 1 & بدون ياسخ & \\
\hline $1 \ldots$ & 0. & جمع & & l.. & 0. & جمع & \\
\hline$T \varepsilon$ & ir & زير جلدى & & 17 & $\wedge$ & آزاد & \\
\hline 1 & r & خوراكى & نوع داروى آهن زدا & $v \varepsilon$ & rv & بيكار & \\
\hline$v \cdot$ & ro & هردو & & $\wedge$ & $\varepsilon$ & خانهدار & | ل شغل \\
\hline $1 .$. & 0. & جمع & & $r$ & 1 & بدون ياسخ & \\
\hline vı & $r q$ & بله & مصرف دارو به صورت & $1 .$. & 0. & جمع & \\
\hline rr & 11 & خير & منظم & $\varepsilon$ & r. & ضعيف & \\
\hline $1 \ldots$ & 0. & جمع & & r & 11 & متوسط & وضعيت \\
\hline or & rY & بله & سابقه ابتلا به بيمارى & 17 & $\wedge$ & خوب & |قتصادى \\
\hline$\varepsilon \wedge$ & $r \varepsilon$ & خير & ديخر & $\wedge$ & $\varepsilon$ & عالى ل & \\
\hline $1 \ldots$ & 0. & جمع & & $1 \ldots$ & 0. & جمع & \\
\hline
\end{tabular}

جدول شماره r: توزيع سطح اضطراب قبل و بعد از آموزش مبر بمان توسط همتا در بيماران مبتلا به تالاسمى مازور آندي

\begin{tabular}{|c|c|c|c|c|}
\hline \multicolumn{2}{|c|}{ بس آزمون } & \multicolumn{2}{|c|}{ يِي آزمون } & \multirow{2}{*}{ سطح اضطر مرحله } \\
\hline درصد & فراوانى & درصد & 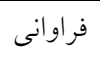 & \\
\hline v. & ro & u & $r \varepsilon$ & خفيف \\
\hline rt & 11 & Y & ir & متوسط \\
\hline$\wedge$ & $\varepsilon$ & 7 & r & 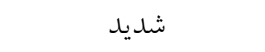 \\
\hline $1 \ldots$ & 0. & $1 .$. & 0. & جمع \\
\hline \multicolumn{2}{|c|}{$I V / \cdot V \pm 11 / \Gamma T$} & \multicolumn{2}{|c|}{$I V / N T \pm 11 / T r$} & انحراف معيار 土 ميانكين \\
\hline $\mathrm{t}=\cdot / \mathrm{N}$ & & & $=\cdot / \varepsilon \mu \varepsilon$ & نتايج آزمون تى زوجى \\
\hline
\end{tabular}

ميانخين و انحراف معيار نمره اضطراب در مراحل بيش

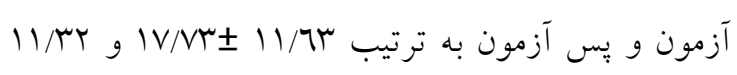
IV/ •VI

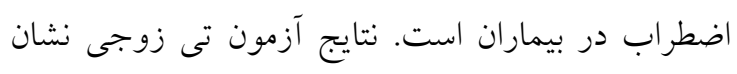

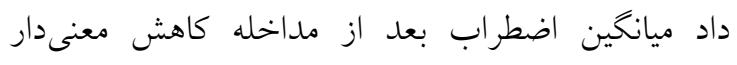

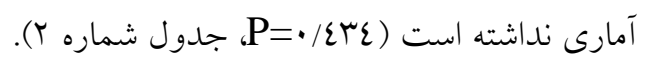


با مرورى بر مطالعات مىتوان جنين نتيجه گيرى كرد كه

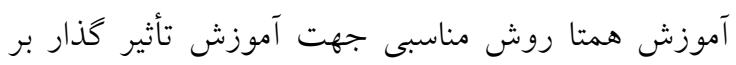

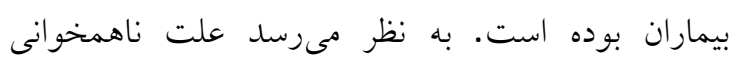

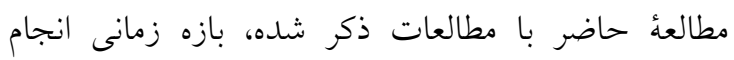

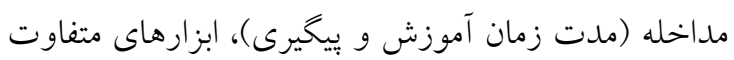
جهت سنجش اضطراب و اختلاف در نوع بيمارى در مطالعات باشد. در مطالعه حاضر قبل از انجام مداخله سطح اضطراب اكثر بيماران خفيف بود. در اين راستا

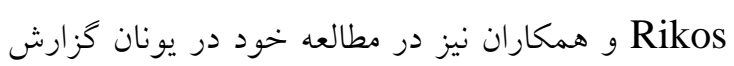

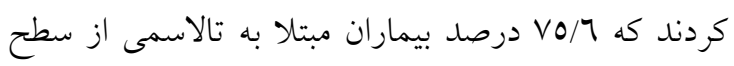

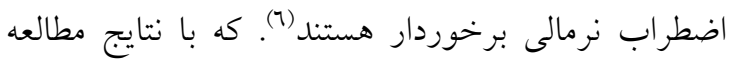

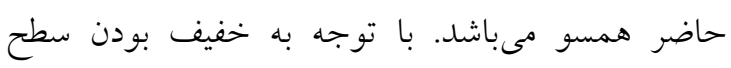

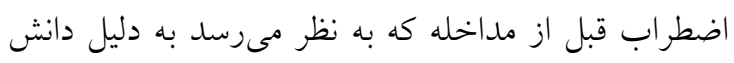

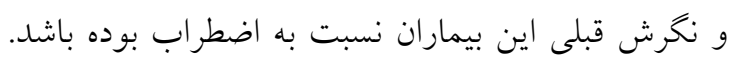

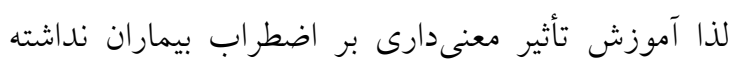

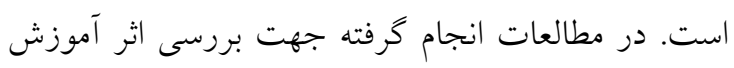

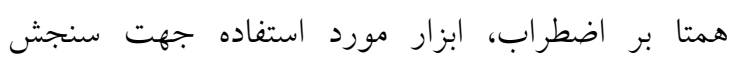

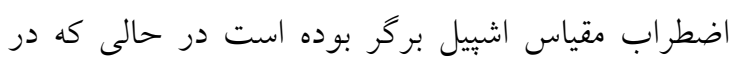

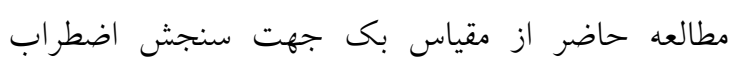
استفاده شد كه اين خود مىتواند از دلايل تفاوت در نتايج

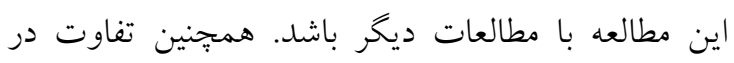

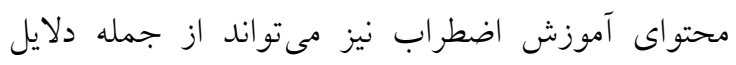

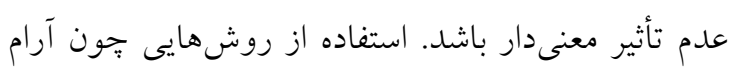

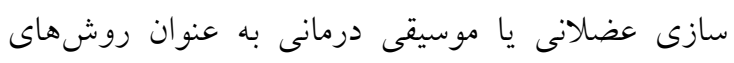

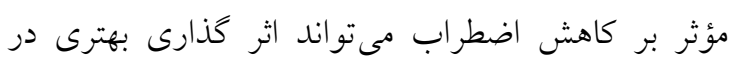
كنار آموزش مهارتهاى كاهش اضطراب داشته باشد (.ع.). از طرفى همزمانى انجام اين مداخله با شيوع همه كيرى كارئ

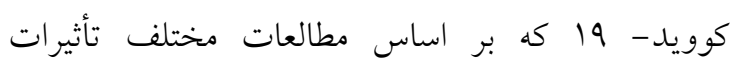

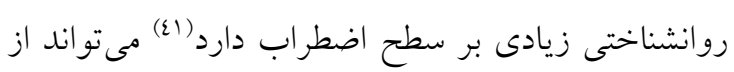
دايل عدم تأثير معنى دار اين آموزش باشئ باشد.

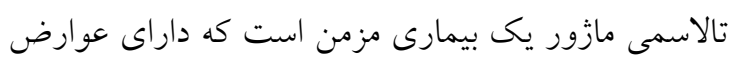

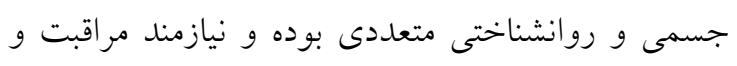

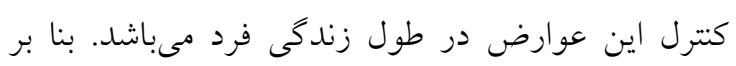

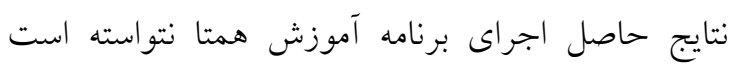

\section{بحث و نتيجه كيرى}

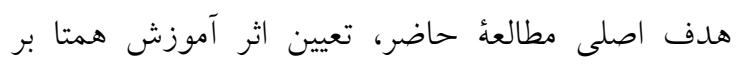

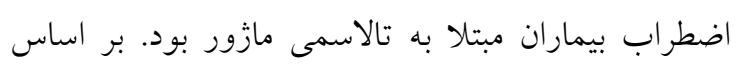

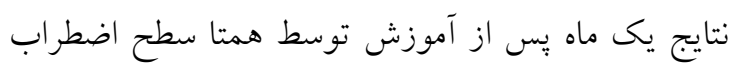

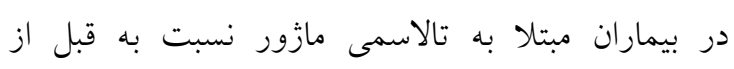

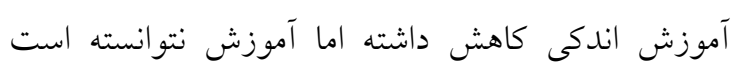
باعث بهبود معنىدار اضطراب در اين بيماران شود. به اله

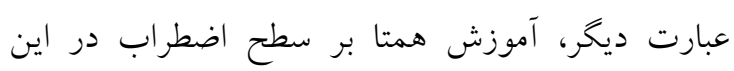
بيماران تأثير مثبت نداشته است. بر اساس مطالعاتى كه

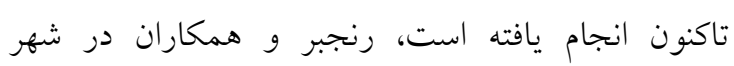

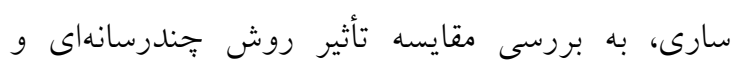

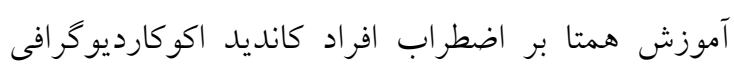

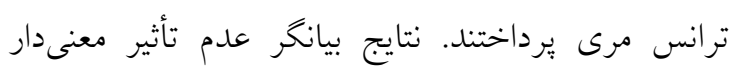

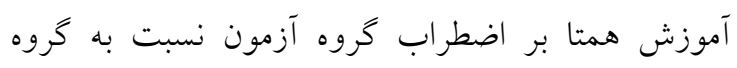
كنترل بود (T) كه با نتايج مطالعه حاضر همسو استر است. همجنين ملازم و همكاران نيز در مطالعه خود كه در شهر

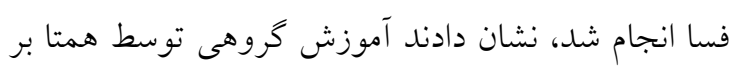
افسردگى بيماران تحت آنزيوگرافى عروق كرونر تأثير نانيان

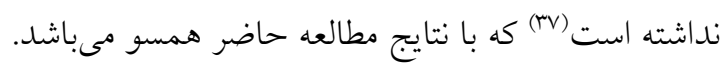

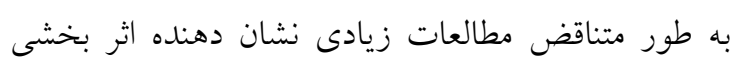

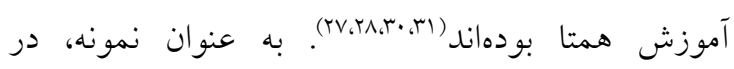

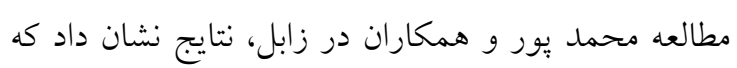
آموزش همتا محور در مقايسه با آموزش برستار به طور

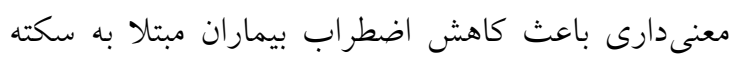

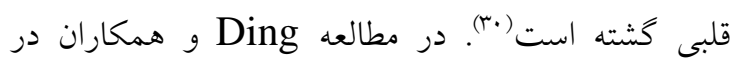
جين نتايج نشان دهنده تأثير آموزش همتا بر كاهش افسردگى، اضطراب و بهبود كيفيت خواب در نوجوانان

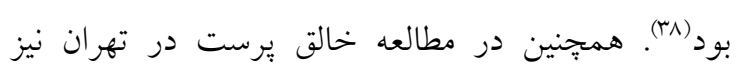

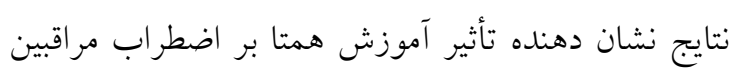

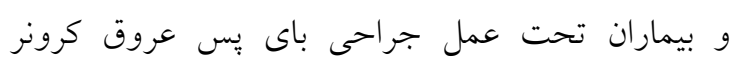

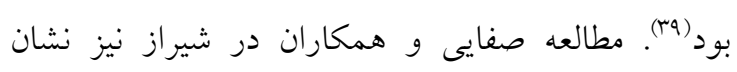
دهنده بهبود خودمراقبتى در بيماران تالاسمى در اثر اين آموزش بود ("1) 


$$
\begin{aligned}
& \text { و تأثيرات روانشناختى بسيارى را در بيى داشت. به علاوه، } \\
& \text { تأثير مثبى بر اضطراب بيماران مبتلا به تالاسمى مازور }
\end{aligned}
$$

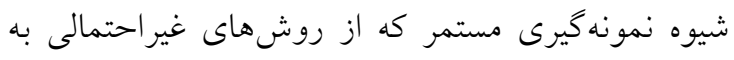

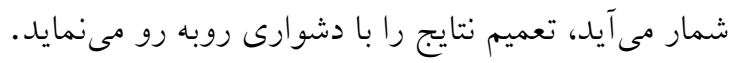

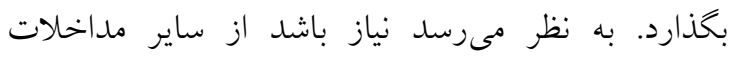

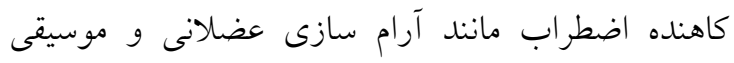

$$
\begin{aligned}
& \text { درمانى در كنار آموزش نيز سود برده شود. همجنين } \\
& \text { تضاد منافع: هيج گونه تعارض منافع توسط نويسندگان }
\end{aligned}
$$

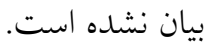

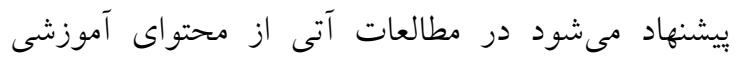

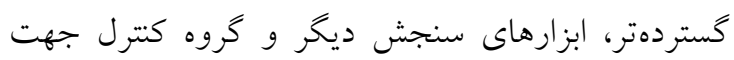

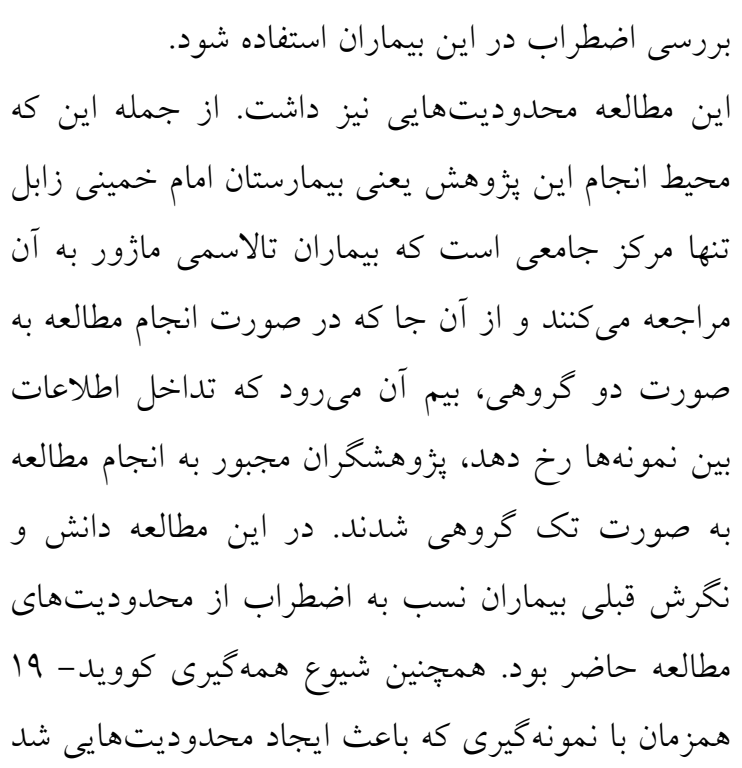

$$
\begin{aligned}
& \text { تقدير و تشكر }
\end{aligned}
$$

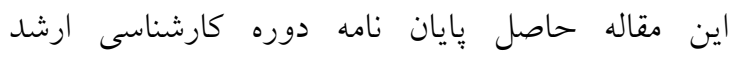

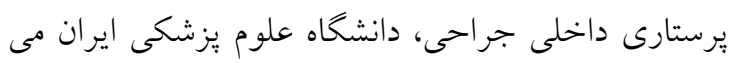

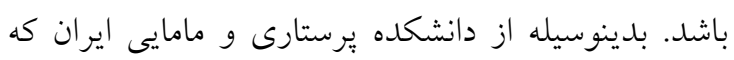

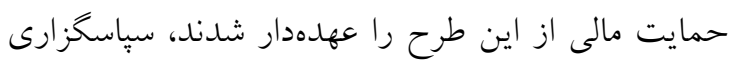

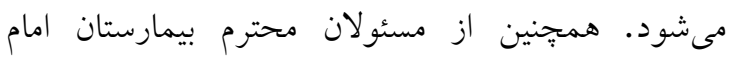

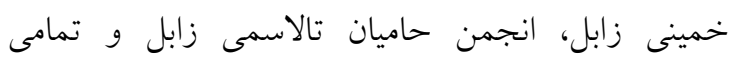

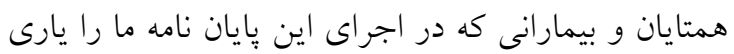

$$
\begin{aligned}
& \text { فرمودند، تقدير و تشكر به عمل مى آيد. }
\end{aligned}
$$

\section{References}

1. Ehsan H, Wahab A, Anwer F, Iftikhar R, Yousaf MN. Prevalence of transfusion transmissible infections in beta-thalassemia major patients in Pakistan: a systematic review. Cureus. 2020;12(8).

2. Raghuwanshi B, Kumari S, Sahoo DP. Clinical and Metabolic Complications in patients with thalassemia undergoing transfusion therapy. J Fam Medic Primary Care. 2020;9(2):973-7.

3. Alsaeed ES, Farhat GN, Assiri AM, Memish Z, Ahmed EM, Saeedi MY, Al-Dossary MF, Bashawri H. Distribution of hemoglobinopathy disorders in Saudi Arabia based on data from the premarital screening and genetic counseling program, 2011-2015. J Epidemiol Global Health. 2018;7:S41-7.

4. Cao A, Kan YW. The prevention of thalassemia. Cold Spring Harb Perspect Med. 2013; 3 (2): a011775.

5. Origa R. $\beta$-Thalassemia. Genet Medic. 2017;19(6):609-19.

6. Rikos N, Giannadaki GK, Spontidaki A, Tzagkaraki M, Linardakis M. Health status, anxiety, depression, and quality of life of patients with thalassemia. J Public Health. 2020 13:1-8.

7. Manzoor MM. Socio-religious Prognosticators of Psychosocial Burden of Beta Thalassemia Major. J Relig Health. 2020;59(6):2866-81.

8. Abolghasemi H, Amid A, Zeinali S, Radfar MH, Eshghi P, Rahiminejad MS, Ehsani MA, Najmabadi H, Akbari MT, Afrasiabi A, Akhavan-Niaki H. Thalassemia in Iran: epidemiology, prevention, and management. J Pediat Hematol/Oncol. 2007;29(4):233-8.

9. Firouzkouhi M, Abdollahimohammad A. Effects of Smartphone Games on Levels of Anxiety, Depression, and Fatigue in Patients with Thalassemia. Journal of Mazandaran University of Medical Sciences. 2020;30(188):62-71. [Persian]

10. Saki N, Dehghani Fard A, Kaviani S, Jalali Far MA, Mousavi SH, Al Ali K, Rahim F. Beta thalassemia: epidemiology and diagnostic and treatment approaches in Iran. Genetics in the $3^{\text {rd }}$ millennium. 2012;10(1):2674-83. [Persian] 
11. Naghibi F, Ahadi H, Tajeri B, Seairafi MR. Effectiveness of Mindfulness-Based Stress Reduction training on psychological symptoms, pain in patients with thalassemia major. Advances in Cognitive Sciences. 2020;22(2):45-53. [Persian]

12. Yengil E, Acipayam C, Kokacya MH, Kurhan F, Oktay G, Ozer C. Anxiety, depression and quality of life in patients with beta thalassemia major and their caregivers. Int J Clin Experim Medic. 2014;7(8):2165-72.

13. Sargolzaei MS, Jahantigh F, Allahyari J. Interventions to improve Quality of Life in Patients with Major Thalassemia: A Systematic Review. Payesh. 2020;19(6):723-33. [Persian]

14. Haji seyedJavadi AR, Shafikhani AA. Evaluation of depression and anxiety in patients with thalassemia: a comparative study. J Qazvin Univ Med Sci. 2017;21(2): 13-21. [Persian]

15. Rafii Z, Ahmadi F, Nourbakhsh SM, Hajizadeh E. The effects of an orientation program on quality of life of patients with thalassemia: A quasi-experimental study. J Caring Sci. 2016;5(3):223-9.

16. Ramazani T, Safarizadeh MH, Alimirzaei R, Rahbarifar S, Fatemi MS. Relationship between quality of life and psychological disorders of patients with thalassemia. Scientific Journal of Iran Blood Transfus Organ. 2015;11(4):362-72. [Persian]

17. Mirghaed MT, Hedayatirad S, Farhadi Z, Sepehrian R, Salemi M, Raeisi P. Correlation of General Health Status of Patients with Major Thalassemia with Demographic Characteristics. $J$. Health Promot. Manag. 2020;9(1):18-24. [Persian]

18. Keşkek ŞÖ, Kırım S, Turhan A, Turhan FG. Depression in subjects with beta-thalassemia minor. Annals of hematology. 2013;92(12):1611-5.

19. Kahrazei F, Hashemzehi S, Rigi Kooteh B. The effect of cognitive behavioral therapy on components of anxiety (physical, behavioral and cognitive) in patients with thalassemia major. Middle Eastern Journal of Disability Studies. 2017;7:1-7. [Persian]

20. Maheri A, Sadeghi R, Shojaeizadeh D, Tol A, Yaseri M, Rohban A. Depression, anxiety, and perceived social support among adults with beta-thalassemia major: cross-sectional study. Korean J Fam Medic. 2018;39(2):101-7.

21. Zolaly MA, Zolaly FM, Al Belowi L, Shuqdar R, Al Belowi Sr MA, Alwasaidi TA, Albadrani M. Depression, Anxiety, and Stress Symptoms in Patients With Beta Thalassemia Major in Almadinah Almunawwarah, Saudi Arabia. Cureus. 2020;12(11):e11367.

22. Yahia S, El-Hadidy MA, El-Gilany AH, Anwar R, Darwish A, Mansour AK. Predictors of anxiety and depression in Egyptian thalassemic patients: a single center study. Int J Hematol. 2013;97(5):604-9.

23. Wang Q, Dong L, Jian Z, Tang X. Effectiveness of a PRECEDE-based education intervention on quality of life in elderly patients with chronic heart failure. BMC cardiovascular disorders. 2017;17(1):1-7.

24. Mohammadi N, Tizhoosh M, Seyedoshohadaei M, Haghani H. Face-to-Face Education vs. Group education on knowledge and anxiety of patients undergoing coronary angiography. Hayat. 2012;18(3):44-53. [Persian]

25. Mohammadpour M, Mohammadpour A, Najafi S. Comparison of the Effect of Peer-led Education and Education by the Healthcare Personnel on Awareness, Attitude and Performance of Lettered People toward Organ Donation. Journal of Babol University of Medical Sciences. 2019;21(1):306-13. [Persian]

26. Edraki M, Zarei A, Soltanian M, Moravej H. The Effect of Peer Education on Self-Care Behaviors and the Mean of Glycosylated Hemoglobin in Adolescents with Type 1 Diabetes: A Randomized Controlled Clinical Trial. Int J Commun Based Nurs Midwif. 2020 Jul;8(3):209.

27. Mohammadpourhodki R, Bagheri H, Basirinezhad MH, Ramzani H, Keramati M. Evaluating the effect of lifestyle education based on peer model on anxiety in patients with acute myocardial infarction. J Complemen Integr Medic. 2019;16(3).

28. Ebrahimi H, Abbasi A, Bagheri H, Basirinezhad MH, Shakeri S, Mohammadpourhodki R. The role of peer support education model on the quality of life and self-care behaviors of patients with myocardial infarction. Patient Educ Couns. 2021;104(1):130-5.

29. Or KY, Yip BH, Lau CH, Chen HH, Chan YW, Lee KP. Peer education group intervention to reduce psychological insulin resistance: a Pilot mixed-method study in a Chinese population. Diabetes Therapy. 2018;9(1):113-24. 
30. Mohammadpoor HR, Rahnama M, Abdollahimohammad A, SHAHRAKI VA, Shaamsizadeh M. A comparison of the effect of training by peers and nurses on anxiety in patients with myocardial infarction: a randomized control trial. PCNM. 2016;6(3):12-23. [Persian]

31. Safaei S, Abedi H, Parand S, Karimi M. Evaluation of the Effect of Support-Training System of Peer Group on Promotion of Self-Care in $\beta$-Thalassemia Major Patients in Southern Iran. Hemoglobin. 2019;43(3):198-203.

32. Webel AR, Okonsky J, Trompeta J, Holzemer WL. A systematic review of the effectiveness of peer-based interventions on health-related behaviors in adults. Am $J$ Public Health. 2010;100(2):247-53.

33. Beck AT, Steer RA, Carbin MG. Psychometric properties of the Beck Depression Inventory: Twenty-five years of evaluation. Clin Psychol Rev. 1988;8(1):77-100.

34. Kaviani H, Mousavi AS. Psychometric properties of the Persian version of Beck Anxiety Inventory (BAI). Tehran University Medical Journal. 2008;66(2):136-40. [Persian]

35. Kheftan P, Eghlima M, Sepiddam M. Stress management techniques and its effects on health promotion. Quarterly Journal of Social Work. 2015 10;4(3):18-27. [Persian]

36. Lateh ZR, Jafari H, Mohammadpour RA, Jalalian R, Nikpajouh A, Esmaeili R. Comparison the effect of multimedia and peer training methods on the anxiety of Trans Esophagus Echocardiography candidate. J Nurs Midwif Sci. 2019;6(1):1.

37. Molazem Z, Shahabfard Z, Askari A, Kalyani MN. Effects of a peer-led group education on fear, anxiety and depression levels of patients undergoing coronary angiography. Investigacion y educacion en enfermeria. 2018;36(1):e13.

38. Ding X, Yao J. Peer Education Intervention on Adolescents'anxiety, Depression, and Sleep Disorder during the COVID-19 Pandemic. Psychiatria Danubina. 2020;32(3-4):527-35.

39. Khaleghparast S, Heydarpoor S, Salesi M, Totonchi M. The effect of peer education on the anxiety-of post Coronary Artery Bypass Graft patients and caregivers in Rajaee Cardiovascular Medical and Reseach Center. Iranian Journal of Cardiovascular Nursing. 2018 10;7(3):24-9. [Persian]

40. Wilczyńska D, Łysak-Radomska A, Podczarska-Głowacka M, Zajt J, Dornowski M, Skonieczny P. Evaluation of the effectiveness of relaxation in lowering the level of anxiety in young adults-a pilot study. Int J Occup Med Environ Health. 2019;32(6):817-24.

41. Liu S, Yang L, Zhang C, Xiang YT, Liu Z, Hu S, Zhang B. Online mental health services in China during the COVID-19 outbreak. The Lancet Psychiatry. 2020;7(4):e17-8. 